\title{
Development of a new recombinant p24 ELISA system for diagnosis of bovine leukemia virus in serum and milk
}

\author{
Lanlan Bai ${ }^{1,2} \cdot$ Kana Yokoyama $^{1,2} \cdot$ Sonoko Watanuki ${ }^{1,2,3} \cdot$ Hiroshi Ishizaki $^{5} \cdot$ Shin-nosuke Takeshima ${ }^{1,2,4}$. \\ Yoko Aida ${ }^{1,2,3,4}$
}

Received: 18 May 2018 / Accepted: 20 August 2018 / Published online: 11 October 2018

(c) Springer-Verlag GmbH Austria, part of Springer Nature 2018

\begin{abstract}
Bovine leukemia virus (BLV) is a retrovirus that causes enzootic bovine leucosis. Here, we designed a p24 enzyme-linked immunosorbent assay (ELISA) to detect antibodies specific for BLV capsid protein p24 (encoded by the gag gene) in bovine serum samples. The $p 24$ gene was inserted into an Escherichia coli expression system, and recombinant proteins (GST-p24, p24, and His-p24) were purified. His-p24 was the most suitable antigen for using in the ELISA. The cut-off point was calculated from a receiver operating characteristic curve derived from a set of 582 field samples that previously tested positive or negative by BLV-CoCoMo-qPCR-2, which detects BLV provirus. The new p24 ELISA showed almost the same specificity and sensitivity as a commercial gp51 ELISA kit when used to test field serum samples, and allowed monitoring of p24 antibodies in raw milk and whey. Comparing the results for the p24 ELISA and gp51 ELISA revealed that p24 antibodies were detected earlier than gp51 antibodies in three out of eight calves experimentally infected with BLV, indicating improved detection without diminishing BLV serodiagnosis. Thus, the p24 ELISA is a robust and reliable assay for detecting BLV antibodies in serum or milk, making it is a useful tool for large-scale BLV screening.
\end{abstract}

\section{Introduction}

Bovine leukemia virus (BLV) is the causative agent of enzootic bovine leucosis (EBL), a chronic lymphoproliferative neoplastic disease of cattle [1-3,54]. The virus is closely

Handling Editor: Chan-Shing Lin.

Yoko Aida

aida@riken.jp

1 Nano Medical Engineering Laboratory, RIKEN

Cluster for Pioneering Research, 2-1 Hirowasa, Wako,

Saitama 3510198, Japan

2 Viral Infectious Disease Unit, RIKEN, 2-1 Hirosawa, Wako, Saitama 3510198, Japan

3 Laboratory of Global Animal Resource Science, Department of Global Agriculture Science, Graduate School of Agriculture and Life Science, The University of Tokyo, 1-1-1 Yayoi, Bunkyo-ku, Tokyo 1138657, Japan

4 Laboratory of Viral Infectious Diseases, Department of Computational Biology and Medical Sciences, Graduate School of Frontier Science, The University of Tokyo, 1-1-1 Yayoi, Bunkyo-ku, Tokyo 1138657, Japan

5 Grazing Animal Unit, Division of Grassland Farming, Institute of Livestock and Grassland Sciences, NARO, 768 Senbonmatsu, Nasushiobara, Tochigi 3292793, Japan related to human T-cell leukemia viruses type 1 and $2[3$, 14]. BLV has spread worldwide [40-43, 45, 50]; indeed, BLV seropositivity is detected in $40.5 \%$ of dairy herds in Japan [33], 78\% of dairy herds in Canada [34], and 79.5\% of dairy herds in the USA [4].

BLV is a major animal health problem causing digestive disturbances, loss of appetite, weight loss, weakness or general debility and sometimes neurological manifestations [37], leading to huge economic losses worldwide [36, 38]. Previously, the economic losses were estimated only in terms of animal health, semen intended for export [28], and a low percentage of BLV-infected cattle that develop B-cell lymphoma [3]. However, a recent study reported that BLVpositive cows produce $218 \mathrm{~kg}$ less milk than BLV-negative healthy cows [38], indicating that even subclinical forms of the disease can cause economic losses by reducing yields $[45,55]$ and longevity $[5,11]$, and increasing morbidity due to weakened immune responses [14, 39]. Rhodes et al. reported estimated economic losses of $\$ 412$ per case of lymphosarcoma [45].

BLV comprises structural proteins encoded by the gag gene matrix protein $\mathrm{p} 15$, capsid protein $\mathrm{p} 24$ (the most abundant capsid protein), and nucleocapsid protein $\mathrm{p} 12$ [26, 52]. The envelope proteins (surface glycoprotein [gp51] and transmembrane 
protein [gp30]) are encoded by the env gene. Upon infection by BLV, cattle generate antibodies directed primarily against viral proteins p24 and gp51 [6].

BLV is diagnosed by the detection of viral antibodies and viral antigens [43]. During natural or iatrogenic transmission of BLV, one of the earliest indications of infection is onset of a humoral anti-viral response (at about 1-8 weeks post-inoculation) $[25,44]$. The most commonly used serological tests are radioimmunoassay, the agar gel precipitation test (AGID), phytohemagglutinin (PHA) tests, and enzyme-linked immunosorbent assays (ELISAs) [18, 43]. The AGID and PHA methods can yield variable results due to different sensitivities and specificities. In addition, AGID is less sensitive and can miss infected cattle harboring a low antibody titer [9, 31] whereas ELISA is more sensitive and specific [47]. Most commercially available ELISAs are designed to detect antibodies specific for gp51 [18, 23]. For example, the gp51 antigen for the commercial ELISA kit used in Japan is purified from inactivated and solubilized virus obtained from persistent BLVinfected sheep fetal kidney cells using monoclonal antibody which makes gp51 production a rather difficult, expensive, and time-consuming process. A previous study indicated that both the immunogenicity and concentration of retroviral capsid proteins in virions and infected cells are higher than those of gp51 [44]. Furthermore, p24 antibodies present in most animals during the early period post-BLV infection [41]. A certain number of field infected cattle have p24 antibodies at high levels [22, 30], and tests targeting BLV p24 antigen test as well as gp51 antigen are approved by the World Organization for Animal Health (OIE) [37]. In addition, p24 antibody is an important marker of response for the virus-like particle (VLP) vaccine (VLP: assembled by BLV gag gene [19, 20]) in immunized animals that are negative using gp51 ELISA. The Gag p24 protein is also widely used as a surrogate marker for diagnosis of HIV-1, HTLV-1, HTLV-II, and STLV-I infection [12, 46, 48, 51].

Recently, recombinant proteins have become widely used in immunoassays for detecting antibodies [13, 32]. Therefore, we expressed the BLV p24 protein in Escherichia coli (E. coli) and used the purified product as an antigen to develop a p24 ELISA to detect anti-BLV antibodies. This fast, simple, specific, and low cost p24 ELISA assay is a robust alternative to the currently available commercial kit used to screen for BLV diagnosis, and will be useful for future vaccine development such as VLP or a subunit p24 immunogen.

\section{Materials and methods}

\section{Animals, serum, genomic DNA, milk, and whey}

BLV-negative three Holstein calves, two Jersey calves, and three Japanese Black calves were inoculated with white blood cells containing more than $10^{6}$ proviral copies of BLV (isolated from naturally infected cattle). Serum samples were obtained at weekly intervals over a period of 12 weeks post-inoculation.

Blood samples (in EDTA) were also collected from 582 cattle from seven farms in four provinces in Japan. Serum was obtained (tube containing clot activator and serum separator gel), and genomic DNA was isolated using the Wizard Genomic DNA Purification Kit (Promega Corporation, Tokyo, Japan). BLV-positive serum which was designated as EBL serum was isolated from cattle that develop B cell lymphoma and used as a positive control for all tests. Raw milk was obtained from 194 cows that were diagnosed for BLV infection by ELISA using gp51 antibodies and p24 antibodies for testing serum samples: 63 were BLV-negative and 131 were BLV-positive. Milk was centrifuged at 20400 $\times \mathrm{g}$ for 30 min to obtain whey. All samples were stocked at $-20^{\circ} \mathrm{C}$ until use.

\section{Construction of the p24 plasmid}

The $p 24$ gene was amplified from an infectious molecular clone, pBLV-IF [16], using primer set p24-EcoRI-F, 5'-AAGAATTCCTTGCCAATCATATCTGAAGGG-3' and p24-XhoI-R, 5'-TTCTCGAGTCAGAGAACTGCAGGCTG TTTCACC-3'. The EcoRI and XhoI sites are underlined. The $648 \mathrm{bp}$ PCR product was subcloned into the EcoRI and XhoI sites of the entry vector pGEX-6p-3 (GE Healthcare, Uppsala, Sweden) and designated glutathione S-transferase (GST)-p24/pGEX-6p-3. To yield His-p24/pGEX-6p-3, the GST tag of GST-p24/pGEX-6p-3 was replaced with a $6 \times$ histidine (6-His) tag using primer set His-p24-F, 5'-ATGCAT CATCACCATCACCACTCGGATCTGGAAGTTCTG-3' and His-p24-R, 5'-GTGGTGATGGTGATGATGCATGAA TACTGTTTCCTGTGT-3 (the 6-His tag is indicated in double underlined). All of the plasmids were confirmed by sequencing.

\section{Expression and purification of recombinant proteins}

GST-p24/pGEX-6p-3 and His-p24/pGEX-6p-3 were transformed into E. coli BL21-CodonPlus ${ }^{\circledR}$ (DE3)-RIPL competent cells (Agilent Technologies, Tokyo, Japan), and expression of recombinant proteins were induced by addition of $1 \mathrm{mM}$ isopropyl- $\beta$-d-thiogalactoside at $25^{\circ} \mathrm{C}$ with agitation. The expressed GST-p24 protein was harvested using Glutathione Sepharose ${ }^{\text {TM }} 4$ Fast Flow (GE Healthcare Bio-Sciences, Uppsala, Sweden), according to the manufacturer's instructions, and concentrated using a centrifugal filter column (Amicon ${ }^{\circledR}$ Ultra-15; Merck Millipore Ltd., Darmstadt, Germany). The GST-p24 was digested with a precision protease to release the GST tag. The His-p24 protein was purified using a His GraviTrap column (GE Healthcare Life Sciences), according 
to the manufacturer's instructions. The concentration of each recombinant protein was measured using a bicinchoninic acid protein kit (ThermoFisher Science, Waltham, MA).

\section{Detection of recombinant proteins}

Purified recombinant GST-p24, p24, His-p24, and GST proteins were subjected to sodium dodecyl sulfate polyacrylamide gel electrophoresis (SDS-PAGE) on $12 \%$ gels, which were then stained with Coomassie brilliant blue (CBB). The proteins were transferred to a membrane and blocked with $5 \%$ skim milk in phosphate-buffered saline (PBS)-T buffer at room temperature. After washing three times, the membrane was incubated overnight at $4^{\circ} \mathrm{C}$ with an anti-BLV p24 monoclonal antibody (Mab) (BLV-3; VMRD, Pullman, WA), an anti-GST polyclonal antibody (Pab) (GE Healthcare Life Sciences), or an anti-His tag Mab [HRP] (GenScript, Piscataway Township, $\mathrm{NJ}$ ), followed by either peroxidase-conjugated affinipure goat anti-mouse IgG (Jackson ImmunoResearch Inc., West Grove, PA) or horseradish peroxidase-conjugated anti-goat IgG (Santa Cruz Biotechnology, Texas, TX).

\section{Development of a new recombinant p24 protein ELISA system}

To determine the optimal concentrations of GST-p24, p24, and His-p24 required for immobilization, recombinant proteins were serially diluted 2-fold from 40 to $0.156 \mu \mathrm{g} / \mathrm{ml}$ in 0.1 $\mathrm{M} \mathrm{NaHCO}_{3}$ buffer ( $\mathrm{pH}$ 9.0) and coated onto multiwell plates (Sumitomo Bakelite Co., Ltd., Tokyo, Japan) overnight at $4^{\circ} \mathrm{C}$. The coated plates were washed with PBS-T buffer and applied with the anti-BLV p24 Mab (BLV-3) or with serum from BLVfree cattle or cattle experimentally infected with BLV (samples were diluted in sample dilution buffer [7.20 mM Na $2 \mathrm{HPO}_{4}$ $12 \mathrm{H}_{2} \mathrm{O}, 2.82 \mathrm{mM} \mathrm{NaH}{ }_{2} \mathrm{PO}_{4} 2 \mathrm{H}_{2} \mathrm{O}, 150.24 \mathrm{mM} \mathrm{NaCl}, 1 \%$ tween 20, and 5\% skim milk]) and incubated with antigens for $1 \mathrm{~h}$ at $37^{\circ} \mathrm{C}$. The plates were then washed and incubated at $37^{\circ} \mathrm{C}$ for $30 \mathrm{~min}$ with HRP-conjugated goat anti-mouse IgG (Amersham Bioscience, Piscataway, NJ) or peroxidase-conjugated goat anti-bovine IgG (Jackson ImmunoResearch Inc.). Finally, 3,3',5,5' -tetramethylbenzidine (TMB) substrate was applied to the plates and the reaction was stopped by addition of $1 \mathrm{~N} \mathrm{H}_{2} \mathrm{SO}_{4}$. Optical density (OD) values were read, and the status of a sample that are evaluated by the sample to positive ratio (S/P ratio) was calculated as follows:

$\frac{\mathrm{S}}{\mathrm{P}}=\frac{\text { Sample }(\text { antigen }- \text { no antigen })-\text { Blank }}{\text { Positive control(antigen }- \text { no antigen })- \text { Blank }}$

The cut-off point for the S/P value was calculated from receiver operating characteristics (ROC) curves.

To analyze the sensitivity and specificity of the assay, a series of dilutions of serum E4 (the European Community's reference serum), EBL serum (isolation from BLV-infected cattle with B cell lymphoma) and BLV-negative serum were evaluated by p24 ELISA.

In addition, the collected 582 serum samples, serum from eight experimentally infected calves and $194 \mathrm{milk} /$ whey samples were analyzed.

\section{Measurement of BLV proviral load}

BLV proviral load (PVL) in all the DNA samples (the collected 582 samples and 8 experimentally infected claves) was measured using the BLV-CoCoMo-qPCR-2 method as described previously [17, 29, 35, 39, 40, 49, 56].

\section{Measurement of gp51 antibodies}

Anti-BLV gp51 antibodies were measured using an antiBLV antibody ELISA kit (JNC, Tokyo, Japan), according to the manufacturer's instructions.

\section{Results}

\section{Preparation of recombinant proteins}

As shown in Fig. 1, the 648 bp fragment of p24 gene encoding the BLV Gag capsid protein p24 was inserted into pGEX-6p-3 with an N-terminal GST or His tag. The calculated (using Compute $\mathrm{pI} / \mathrm{Mw}$ tool) molecular weights of GST-p24, p24, GST, and His-p24 were 50, 24, 26, and 25 KDa, respectively; a single band with the correct size for each recombinant protein was detected by $\mathrm{CBB}$ staining (Fig. 1A, bottom). Western blotting with an anti-p24 Mab, an anti-GST Pab, and an anti-His Mab detected p24 (GSTp24, p24, and His-p24), GST-p24 and GST, and His-p24, respectively (Fig. 1A, bottom). This indicated that the target proteins were expressed and produced correctly.

\section{Development of a new p24 ELISA assay}

As shown in Fig. 1B, p24, GST-p24, and His-p24 reacted specifically with the anti-BLV p24 Mab and with BLV-positive serum. An anti-BLV p24 Mab reacted strongly with antigens, while the optimal concentrations of p24, GST-p24, and His-p24 for immobilization on the ELISA plate were 5, 2.5 , and $2.5 \mu \mathrm{g} / \mathrm{ml}$, respectively. Likewise, each peak reaction value was obtained for BLV-positive bovine serum when the concentrations of p24, GST-p24, and His-p24 were 10, 5, and $5 \mu \mathrm{g} / \mathrm{ml}$, respectively. However, GST-p24 reacted nonspecifically with BLV-negative serum when used at a concentration $>10 \mu \mathrm{g} / \mathrm{ml}$. In contrast, p24 and His-p24 showed specific reactions at high concentrations. Furthermore, when 
A

\begin{tabular}{|c|c|c|}
\hline \multirow[b]{2}{*}{ GST-p24 } & \multicolumn{2}{|c|}{0} \\
\hline & GST & p24 \\
\hline p24 & 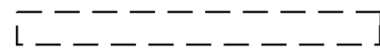 & p24 \\
\hline GST & ${ }^{-}$ & \\
\hline His-p24 & \begin{tabular}{|l|} 
His \\
\end{tabular} & p24 \\
\hline
\end{tabular}

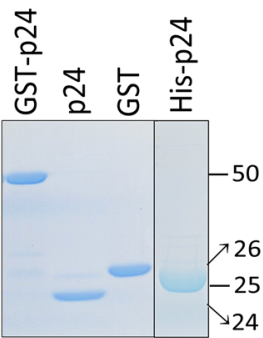

CBB staining

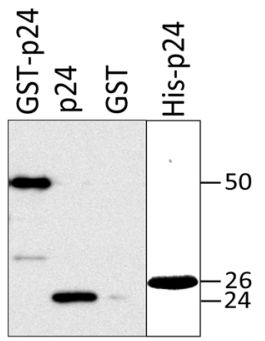

$\alpha-p 24$

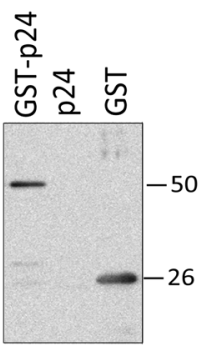

$\alpha$-GST

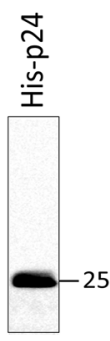

Western blot

B
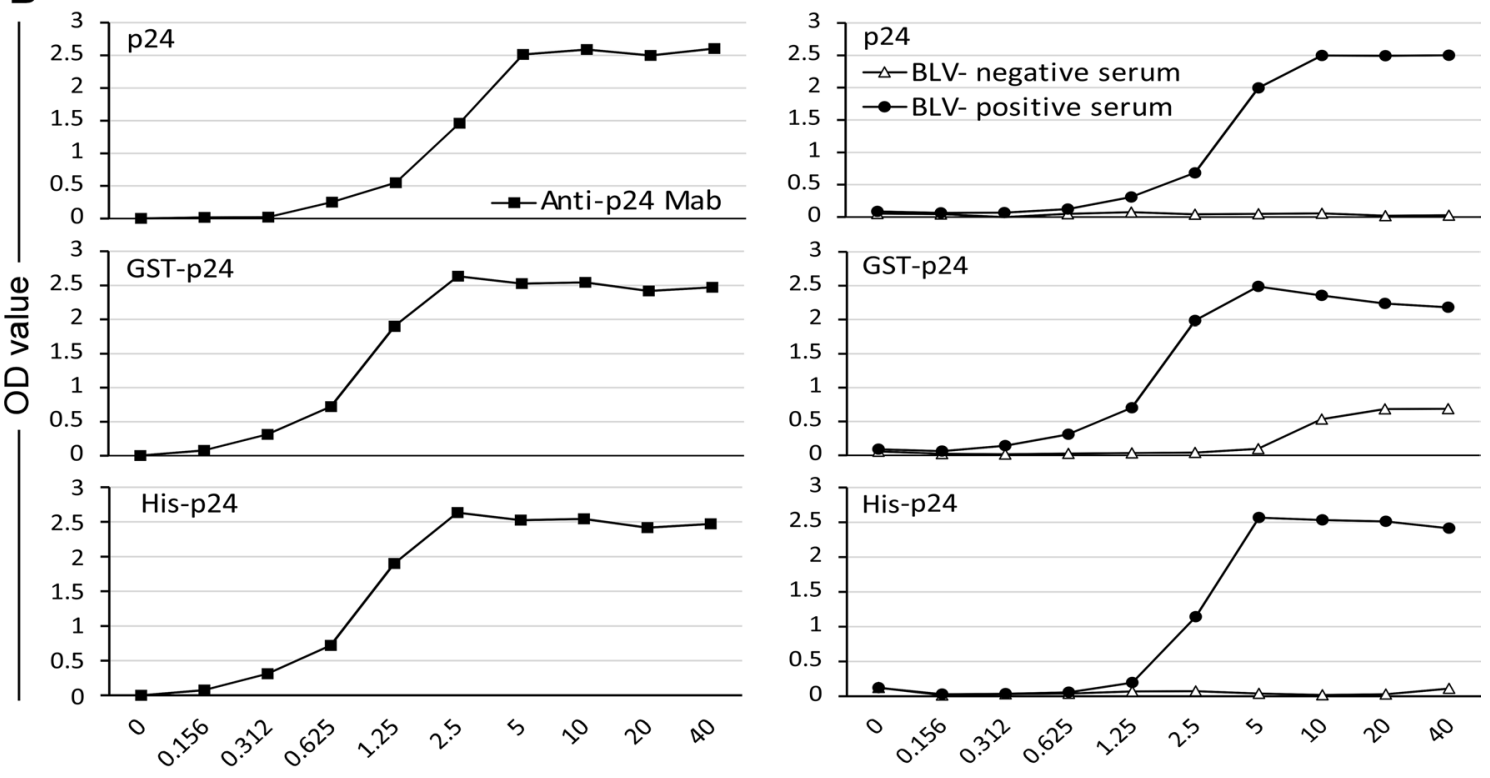

Protein concentration ( $\mu \mathrm{g} / \mathrm{ml}$ )
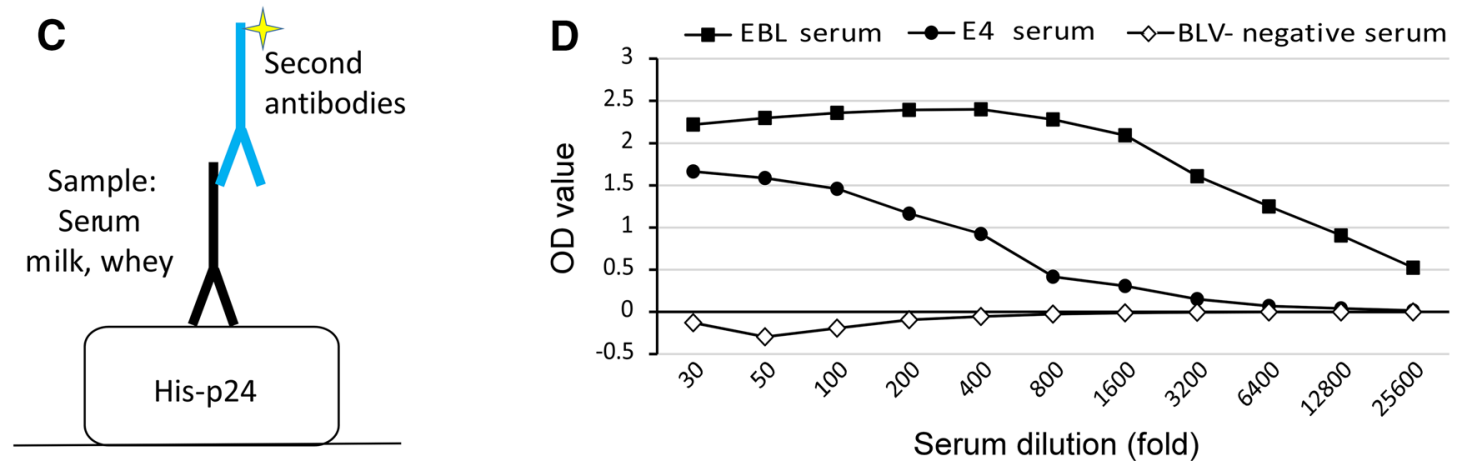
4 Fig. 1 Development of the new p24 ELISA system. (A) Schematic showing the BLV recombinant fusion proteins: Glutathione S-Transferase (GST)-p24, p24, GST (left upper), and His-p24 (right upper). The p24 protein is shown in gray and comprises amino acids 1-216. Proteins of the correct size are visible on coomassie brilliant blue (CBB) stained gels and on western blots detected using antibodies specific for p24, GST, and His (bottom). (B) Optimization of p24, GST-p24, and His-p24 concentrations used to coat the plate wells. Recombinant proteins were serially diluted 2-fold and coated. Plates were incubated with either an anti-p24 Mab (left) or BLV-negative/ positive cattle serum (right) prior to measurement of OD values. (C) Schematic representation of the new recombinant p24 ELISA system. Purified His-p24 protein was immobilized on plate. Serum samples were added to response and the antigen-antibody complex was detected using horseradish peroxidase (HRP)-conjugated anti-bovine $\mathrm{IgG}$. After washing, the wells were incubated with substrate solution and the reaction was stopped with $\mathrm{H}_{2} \mathrm{SO}_{4}$ prior to reading of optical density. (D) Analysis of sensitivity and specificity of p24 ELISA. The European Community's reference serum E4, EBL serum (isolation from BLV-infected cattle with B cell lymphoma) and BLV negative serum were diluted into a series to detected p24 antibodies by p24 ELISA

used at low concentrations $(2.5-5 \mu \mathrm{g} / \mathrm{ml})$, His-p24 reacted more strongly with BLV-positive bovine serum than 24 .

His-p24 was therefore chosen as the diagnostic antigen for the p24 ELISA. The optimal concentration for immobilization was $5 \mu \mathrm{g} / \mathrm{ml}$. The recombinant p24 ELISA assay is illustrated in Fig. 1C. Furthermore, the data obtained with a series of dilutions of E4 and EBL serum showed that the this p24 ELISA has high sensitivity and specificity (Fig. 1D): E4 was reacted at 1:12800 and EBL serum showed antibody activity of $>1: 26000$. By contrast, control negative serum showed no reaction with His-p24.

\section{Determining the cut-off point for the p24 ELISA system}

The PVL in 582 cattle was measured using BLV-CoCoMoqPCR-2. The results showed that 295 samples were positive and the remaining 287 samples were negative. To determine the cut-off point for the S/P value, ROC curves were constructed to evaluate the potential of antibodies to discriminate between animals with a detectable or undetectable PVL. ROC curve analysis yielded an area under the curve (AUC) of 0.905 , which enables meaningful interpretation of p24 antibody levels in BLV-positive and -negative cattle. From this, the cut-off for the $\mathrm{S} / \mathrm{P}$ value of the new p24 ELISA was calculated as 0.40 (Fig. 2).

\section{Comparison of diagnostic results obtained by the p24 ELISA and other detection methods}

The new p24 ELISA was used to measure anti-BLV antibody levels in 582 samples collected from cattle in the field. First, we compared the specificity and sensitivity of the new p24 ELISA with that of BLV-CoCoMo-qPCR-2 for the diagnosis of BLV infection (Table 1). The results showed that 249/582 cattle (42.8\%) were positive for both PVL and anti-p24 antibodies and that $255 / 582(43.8 \%)$ cattle were negative with both PVL and anti-p24 antibodies. Overall, 295 cattle were positive for PVL, but 46 of them were negative with antip24 antibodies (sensitivity, 84.4\%; 95\% CI, 79.8-88.4\%). Similarly, 287 cattle were negative for PVL, but 32 of them were positive with anti-p24 antibodies (specificity, $88.9 \%$; 95\% CI, 84.6-92.2\%).

Next, we compared the specificity and sensitivity of the new p24 ELISA with those of a commercial gp51 ELISA kit. As shown in Table 2, 266/582 (45.7\%) cattle were positive for both anti-p24 and anti-gp51 antibodies, and 260/582 $(44.7 \%)$ were negative for both anti-p24 and anti-gp51 antibodies. Overall, 307 cattle were positive with anti-gp51 antibodies, but 41 of these were negative with anti-p24 antibodies (sensitivity of $86.6 \%$; 95\% CI, 86.6-90.2\%). Similarly, 275 cattle were negative for with anti-gp51 antibodies, but 15 of these were positive with anti-p24 antibodies (specificity, 94.5\%; 95\% CI, 91.2-96.9\%). Taken together, the data suggest that the new p24 ELISA has high sensitivity and high specificity when compared with BLV-CoCoMoqPCR-2 and the gp51 ELISA kit.

Table 3 summarizes the results obtained using the three different methods. Overall, 249 (42.8\%) cattle were positive for provirus, gp 51 antibodies, and p24 antibodies, and 242 (41.6\%) cattle were negative for provirus, gp51 antibodies, and p24 antibodies. By contrast, 18 cattle were PVL-positive only, 15 were p 24 antibody-positive only, and 13 were gp51 antibody-positive only. Of 17 cattle that were negative for PVL, all had detectable p24 and gp51 antibodies. In addition, 56 samples gave different results in the p24 and gp51 ELISAs, including 28 cattle that were PVL- and gp51-positive, 13 samples that were gp51-positive and PVL-negative, and 15 that were $\mathrm{p} 24$-positive and PVL-negative. This suggests that production of anti-BLV antibodies may vary from cow to cow. According to our data, 277 (93.9\%) of 295 cattle which tested positive for BLV provirus, as determined by BLV CoCoMo-qPCR-2, were found with at least one type of antibodies, and 277 (85.5\%) of 324 cattle which tested positive for antibody had provirus. Combined together, the data suggests that naturally infected cattle produce anti-p24 and anti-gp51 antibodies, but it is unclear which is generated first.

\section{Determination of the period post-infection during which p24 and gp51 antibodies are induced}

To determine the time course of anti-p24 and anti-gp51 antibody production, we measured their concentrations in eight calves experimentally infected with BLV. Serum samples were diluted 50-fold and tested using the p24 ELISA and the gp51 ELISA kit. As shown in Table 4, three Holstein, two Jersey, 


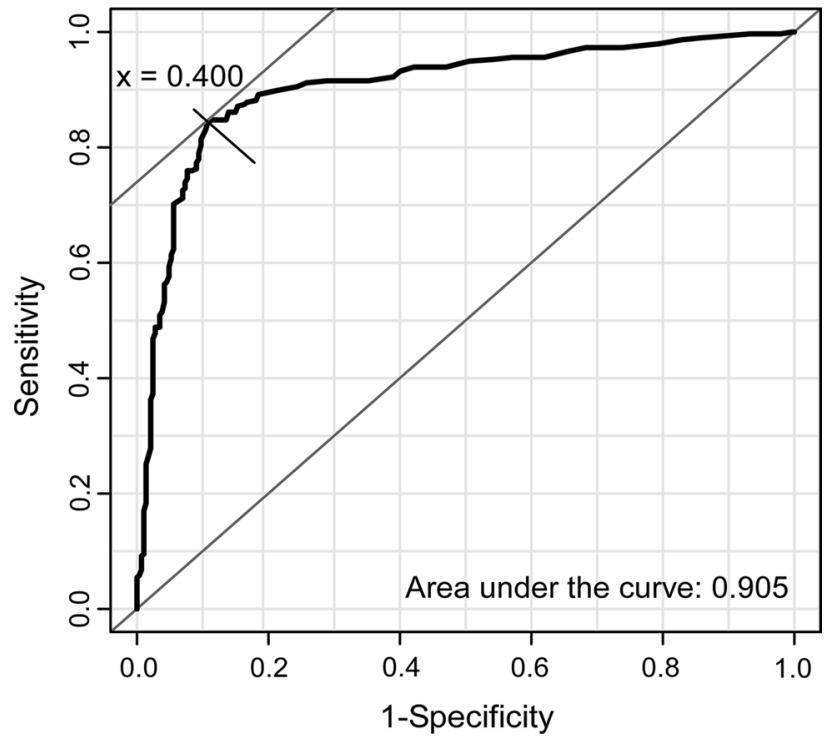

Fig. 2 A receiver operating characteristics (ROC) curve was used to calculate the cut-off point for the S/P value of the new p24 ELISA using serum from 582 cattle that were either positive or negative for BLV (as determined by BLV-CoCoMo-qPCR-2). The cut-off point for the $\mathrm{S} / \mathrm{P}$ value was 0.400 , while the area under the curve was 0.905

Table 1 Testing of 582 samples using the new p24 ELISA and BLVCoCoMo-qPCR-2

\begin{tabular}{lllll}
\hline & & \multicolumn{2}{c}{ BLV-CoCoMo-qPCR-2 } & Total \\
\cline { 2 - 4 } & & Positive & Negative & \\
\hline p24 ELISA & Positive & 249 & 32 & 281 \\
& Negative & 46 & 255 & 301 \\
\multirow{2}{*}{ Total } & 295 & 287 & 582 \\
& & Sensitivity (\%) & Specificity (\%) & \\
& & $(95 \%$ CI $)$ & $(95 \%$ CI $)$ & \\
& 84.4 & 88.9 & \\
& & $(79.8-88.4)$ & $(84.6-92.2)$ & \\
\hline
\end{tabular}

CI, confidence interval

Table 2 Testing of 582 sampes using the new p24 ELISA and the gp51 ELISA kit

\begin{tabular}{lllll}
\hline & & \multicolumn{2}{l}{ gp51 ELISA } & Total \\
\cline { 2 - 4 } & & Positive & Negative & \\
\hline \multirow{2}{*}{ p24 ELISA } & Positive & 266 & 15 & 281 \\
& Negative & 41 & 260 & 301 \\
& & 307 & 275 & 582 \\
& & Sensitivity (\%) & Specificity (\%) & \\
& $(95 \%$ CI $)$ & $(95 \%$ CI $)$ & \\
& 86.6 & 94.5 & \\
& $(82.3-90.2)$ & $(91.2-96.9)$ & \\
\hline
\end{tabular}

CI, confidence interval
Table 3 Detection of BLV in 582 samples using three different methods

\begin{tabular}{lllr}
\hline Provirus $^{\mathrm{a}}$ & ${\mathrm{gp} 51 \mathrm{Abs}^{\mathrm{b}}}^{\mathrm{b}}$ & $\mathrm{p} 24 \mathrm{Abs}^{\mathrm{c}}$ & Head \\
\hline$+^{\mathrm{d}}$ & + & + & 249 \\
+ & + & - & 28 \\
+ & - & + & 0 \\
+ & - & - & 18 \\
- & + & + & 17 \\
- & + & - & 13 \\
- & - & + & 15 \\
- & - & - & 242 \\
\hline
\end{tabular}

${ }^{\mathrm{a}}$ The proviral load (PVL) was measured by BLV-CoCoMo-qPCR-2

${ }^{\mathrm{b}}$ Anti-gp51 antibodies were measured using a gp51 ELISA kit

${ }^{\mathrm{c}}$ Anti-p24 antibodies were measured using the new p24 ELISA

${ }^{\mathrm{d}}+$ : BLV-positive; -: BLV-negative

and three Japanese Black cattle (all BLV-negative and aged $5-12$ months) were inoculated with $10^{6}-10^{7}$ proviral copies (sufficient to induce infection and antibody production). The time of BLV inoculation was defined as week 0. Serum samples were collected weekly up until week 12 . In three calves (N2, KB57, and 587), antibodies specific for p24 were detected earlier than those specific for gp51. In three calves (KB64, KB19, and KB55), anti-p24 and anti-gp51 antibodies were detected simultaneously. Finally, anti-gp51 antibodies were detected earlier than p24 antibodies in two calves (N1 and KB17). Taken together, these results suggest that the BLV p24 protein is a useful marker for diagnosis of BLV infection, since it seems that in many cases p 24 antibodies are detectable earlier than gp51 antibodies.

\section{Detection of p24 antibodies in milk and whey}

Finally, we asked whether the new p24 ELISA could detect antibodies in raw milk and whey (Table 5). The results showed that, of 131 cows with a positive BLV result in blood, 106 showed a positive result in raw milk and whey samples (specificity, 80.9\% [95\% CI, 73.1-87.3\%]). From 63 cattle with BLV-negative blood samples, 58 raw milk samples were negative (sensitivity, 92.1\% [95\% CI, 82.4-97.4\%]). From 43 cattle with BLV-negative blood samples, 37 whey samples were negative (sensitivity, 86.0\% [95\% CI, 72.1-94.7\%]). The gp24 ELISA thus allows a rapid and specific detection of anti-BLV antibodies in milk and whey samples that are easier to obtain in the field than blood samples. 


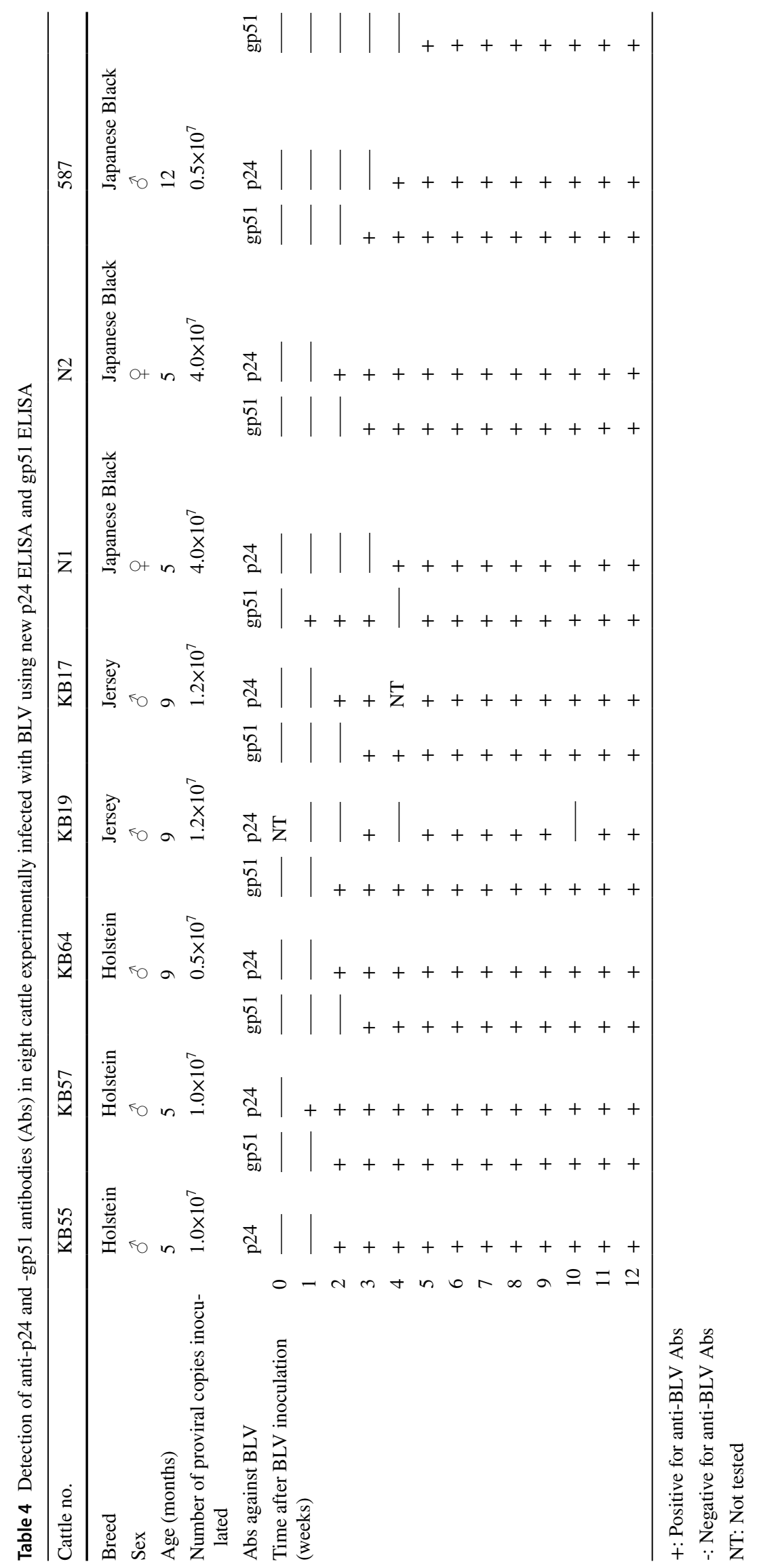


Table 5 Sensitivity and specificity of the new p24 ELISA for detecting BLV in raw milk (194) and whey (174) samples

\begin{tabular}{|c|c|c|c|c|}
\hline & & Blood & & Total \\
\hline & & Positive & Negative & \\
\hline Raw milk & Positive & 106 & 5 & 111 \\
\hline & Negative & 25 & 58 & 83 \\
\hline Total & & 131 & 63 & 194 \\
\hline & & Sensitivity (\%) & Specificity (\%) & \\
\hline & & $(95 \% \mathrm{CI})$ & $(95 \% \mathrm{CI})$ & \\
\hline & & 80.9 & 92.1 & \\
\hline & & $(73.1-87.3)$ & (82.4-97.4) & \\
\hline & & Blood & & Total \\
\hline & & Positive & Negative & \\
\hline Whey & Positive & 106 & 6 & 112 \\
\hline & Negative & 25 & 37 & 62 \\
\hline Total & & 131 & 43 & 174 \\
\hline & & Sensitivity (\%) & Specificity (\%) & \\
\hline & & $(95 \% \mathrm{CI})$ & $(95 \% \mathrm{CI})$ & \\
\hline & & 80.9 & 86.0 & \\
\hline & & $(73.1-87.3)$ & $(72.1-94.7)$ & \\
\hline
\end{tabular}

CI, confidence interval

\section{Discussion}

Here, we showed that (1) of the three examined GST-p24, p24 and His-p24 reagents, the recombinant His-p24 protein was the most suitable one for developing an ELISA system for the rapid and early diagnosis of BLV infection. Larsen et al. have also previously reported that His-p24 gave a very clear reaction with the serum of 16 infected animals [24]. (2) the p24 ELISA showed almost similar sensitivity and specificity for BLV as the commercially available gp51 ELISA kit (although the production of BLV antibodies may vary in individual cattle); (3) p24 antibodies were detected earlier post-infection than gp51 antibodies in three out of eight experimentally infected animals; (4) the p24 ELISA allows monitoring of anti-p24 antibody levels in raw milk and whey. In addition, Gutierrez et al. have also demonstrated that the His-p24-ELISA is a precise assay with good reproducibility [15]. The new p24 ELISA assay has therefore considerable potential for wide scale use.

We detected BLV antibodies and PVL in a large number of samples collected from field cattle. Most PVL-positive samples were also positive for anti-p24 and anti-gp51 antibodies. When cattle are infected with BLV, they generate antibodies specific for the viral p24 and gp51 proteins. Our data showed that infected cattle generate antibodies to both of these proteins. In addition, 17 PVL-negative cattle had detectable anti-p24 and anti-gp51 antibodies, indicating the presence of a recent infection, although not enough provirus was produced to be detectable by
BLV-CoCoMo-qPCR-2). In contrast, 18 cattle were only PVL positive only. This result is supported by the finding of Kelly et al. [21] that using PCR allows for the detection of the provirus as early as 7 days after infection, whereas antibodies can only be detected 3 weeks post infection. Interestingly, only 15 and 13 cattle were positive for either anti-p24 or anti-gp51 antibodies, respectively. It remains unclear which type of antibody appears first in BLV-infected animals because this may depend on the individual animal. Because most field cattle initially have a low PVL, the humoral immune responses are weak. In three of the eight cattle with BLV experimental infection, anti-p24 antibodies were detected earlier than anti-gp51 antibodies although both antibodies were detected at the same time in three other cattle. Radke et al. showed that p24 antibodies were present in most BLV-infected animals very early [44]. In addition, it is possible that retroviral capsid proteins are highly immunogenic and are present in both virions and infected cells at concentrations higher than those of gp51 [44]. However, in two cattle we detected anti-gp51 antibodies earlier than anti-p24 antibodies. The gp51 protein localizes at the surface of the viral particle and since it contains the recognition site for receptors required for virus entry, it is considered to be the natural target for neutralizing antibodies, it may generate a strong immune response in infected cattle [10, 27, 53]. Collectively, these data suggest that a significant proportion of infected cattle may generate anti-p24 antibodies earlier than anti-gp51 antibodies, indicating that the p24 ELISA may improve BLV serodiagnosis and be useful for suppressing virus spread.

Many assays for BLV diagnosis require collection of blood from target cattle [43]. However, collecting milk is less invasive and saves both time and labor. In addition, many false-positive results are obtained for calves below 6 months of age due to ingestion of colostrum, suggesting that anti-BLV antibodies are present in milk. Therefore, we used the p24 ELISA to test samples of raw milk and whey. We detected p24 antibodies in both milk and whey from cattle that had shown positive results with serum. The p24 ELISA is thus a cost-effective method for detecting BLV antibodies in a non-invasive manner.

The gp51 protein is important for the induction of virus neutralizing antibodies, because it contains three conformational epitopes in the N-terminal region of gp51 [7, 8] which is the reason we tried to express the entire gp51 in $E$. coli. However, purifying the entire gp51 was difficult (data not shown) and the purified protein was not recognized by serum from BLV-infected cattle, presumably because major epitopes are nor accessible in recombinant gp51 protein. In contrast, p24 protein has two major epitopes present in the $\mathrm{C}$ terminal region [7, 8]. Furthermore, the recombinant p24 protein expressed in E. coli reacts specifically with p24 
antibodies in serum from BLV infected field serum and with BLV p24 monoclonal antibody making it is a good antigen for BLV detection.

In conclusion, the new p24 ELISA based on a recombinant protein produced on an inexpensive $E$ coli platform is a highly sensitive and specific method for detecting antibodies in serum from both experimentally and naturally infected animals. The assay may soon become available commercially for detecting anti-BLV p24 antibodies in milk and whey and could be valuable for the improved diagnosis of the disease as well as for studying antigens for developing a subunit vaccine or a VLP vaccine.

Acknowledgments We thank Dr. Junko Kohara, Mr. Sunao Hirai, Mr. Susumu Ogawa, Mr. Kenji Mizushiri, Mr. Naoya Takahashi, and Mr. Yoshihiro Okada of the Animal Research Center, Hokkaido Research Organization, for help with experimental infection and drawing of blood. We are also grateful to all members of the Viral Infectious Diseases Field, Nano Medical Engineering Laboratory, RIKEN Cluster for Pioneering Research, for technical assistance, help, and helpful suggestions. We also thank the Support Unit for Biomaterial Analysis, RIKEN BSI Research Resources Center, for help with sequence analysis.

This work was supported by Grants-in-Aid for Scientific Research [A (16H02590) and C (16K02590)] from the Japan Society for the Promotion of Science (JSPS), by a grant from the Project of the NARO Bio-oriented Technology Research Advancement Institution (the special scheme project on regional developing strategy) (Grant No. 16817983), by a grant from the Project of the NARO Bio-oriented Technology Research Advancement Institution (the special scheme project on vitalizing management entities of agriculture, forestry and fisheries) (Grant No. 16930548), and by the Japan Society for the JSPS Postdoctoral Fellowship (Grant No. 16F16404).

\section{Compliance with ethical standards}

Conflicts of interest The authors declare no conflicts of interest.

Animal handling and research ethics All animals were handled by veterinarians from the Animal Research Center in Hokkaido, and RIKEN, Japan in strict accordance with good animal practice following the guidelines of Hokkaido Research Organization and RIKEN. The study was approved by the Animal Ethical Committee and the Animal Care and Use Committee of the Animal Research Center, Hokkaido Research Organization (approval number 1302) and RIKEN Animal Experiments Committee (approval number H29-2-104).

\section{References}

1. Aida Y, Onuma M, Tsukiyama K, Ogawa Y, Fujieda T, Mikami T, Izawa $H$ (1987) Monoclonal antibodies define antigenic regions on the major internal protein p24 of bovine leukemia virus (BLV). Arch Virol 94:315-321

2. Aida Y, Nishino Y, Amanuma H, Murakami K, Okada K, Ikawa Y (1997) The role of tumor-associated antigen in bovine leukemia virus-induced lymphosarcoma. Leukemia 11(Suppl 3):216-218

3. Aida Y, Murakami H, Takahashi M, Takeshima SN (2013) Mechanisms of pathogenesis induced by bovine leukemia virus as a model for human T-cell leukemia virus. Front Microbiol 4:328
4. APHIS (2008) Info sheet: bovine leukosis virus (BLV) on US dairy operations, 2007. US Department of Agriculture Animal and Plant Health Services, Veterinary Services, Center for Epidemiology and Animal Health. http://www.aphis.usda.gov/animal_health/nahms/ dairy/downloads/dairy07/Dairy07_is_BLV.pdf. Accessed 11 Nov 2015

5. Bartlett PC, Norby B, Byrem TM, Parmelee A, Ledergerber JT, Erskine RJ (2013) Bovine leukemia virus and cow longevity in Michigan dairy herds. J Dairy Sci 96:1591-1597

6. Bex F, Bruck C, Mammerickx M, Portetelle D, Ghysdael J, Cleuter Y, Leclercq M, Dekegel D, Burny A (1979) Humoral antibody response to bovine leukemia virus infection in cattle and sheep. Cancer Res 39:1118-1123

7. Bicka L, Kuźmak J, Kozaczyńska B, Plucienniczak A, Skorupska A (2001) Expression of bovine leukemia virus protein p24 in Escherichia coli and its use in the immunoblotting assay. Acta Biochim Pol 48:227-232

8. Bruck C, Portetelle D, Mathot S, Mammerickx M, Burny A (1984) Differential reactivity of cattle and sheep antisera to BLV gp51 epitopes. Leukemia Res. 8:315-321

9. De Boer GF, Boerrigter HM, Akkermans JP, Brenner J (1989) Use of milk samples and monoclonal antibodies directed against BLV-p24 to identify cattle infected with bovine leukemia virus (BLV). Vet Immunol Immunopathol 22:283-292

10. De Giuseppe A, Feliziani F, Rutili D, De Mia GM (2004) Expression of the Bovine leukemia virus envelope glycoprotein (gp51) by recombinant baculovirus and its use in an enzyme-linked immunosorbent assay. Clin Diagn Lab Immunol 11:147-151

11. Erskine RJ, Bartlett PC, Byrem TM, Render CL, Febvay C, Houseman JT (2012) Association between Bovine leukemia virus, production, and population age in Michigan dairy herds. J Dairy Sci 95:727-734

12. Fan P, Li X, Su W, Kong W, Kong X, Wang Z, Wang Y, Jiang C, Gao F (2015) Enhanced sensitivity for detection of HIV-1 p24 antigen by a novel nuclease-linked fluorescence oligonucleotide assay. PLoS One 10:e0125701

13. Faramarzi R, Dolatabadi S (2017) Expression and purification of recombinant HTLV-I/-II linear epitopes antigen and its application for screening of suspected patients. Iran J Microbiol 9:43-49

14. Gillet N, Florins A, Boxus M, Burteau C, Nigro A, Vandermeers F, Balon H, Bouzar AB, Defoiche J, Burny A, Reichert M, Kettmann R, Willems L (2007) Mechanisms of leukemogenesis induced by bovine leukemia virus: prospects for novel anti-retroviral therapies in human. Retrovirology 4:18

15. Gutiérrez G, Alvarez I, Fondevila N, Politzki R, Lomónaco M, Rodríguez S, Dus Santos MJ, Trono K (2009) Detection of bovine leukemia virus specific antibodies using recombinant p24-ELISA. Vet Microbiol 137:224-234

16. Inabe K, Nishizawa M, Tajima S, Ikuta K, Aida Y (1999) The YXXL sequences of a transmembrane protein of Bovine leukemia virus are required for viral entry and incorporation of viral envelope protein into virions. J Virol 73:1293-1301

17. Jimba M, Takeshima SN, Matoba K, Endoh D, Aida Y (2010) BLV-CoCoMo-qPCR: quantitation of bovine leukemia virus proviral load using the CoCoMo algorithm. Retrovirology 7:91

18. Jimba M, Takeshima SN, Murakami H, Kohara J, Kobayashi N, Matsuhashi T, Ohmori T, Nunoya T, Aida Y (2012) BLVCoCoMo-qPCR: a useful tool for evaluating Bovine leukemia virus infection status. BMC Vet Res 8:167

19. Kakker NK, Mikhailov MV, Nermut MV, Burny A, Roy P (1999) Bovine leukemia virus Gag particle assembly in insect cells: formation of chimeric particles by domain-switched leukemia/lentivirus Gag polyprotein. Virology 265:308-318

20. Kakker NK, Mikhailov MV, Jones IM, Roy P (2002) Comparative analysis of the roles of simian immunodeficiency and Bovine 
leukemia virus matrix proteins in Gag assembly in insect cells. Virology 299:48-55

21. Kelly EJ, Jackson MK, Marsolais G, Morrey JD, Callan RJ (1993) Early detection of Bovine leukemia virus in cattle by use of the polymerase chain reaction. Am J Vet Res 54:205-209

22. Kittelberger R, Reichel MP, Meynell RM, Tham KM, Molloy JB (1999) Detection of antibodies against the core protein p24 of the Bovine leukaemia virus in cattle for confirmatory serological testing. J Virol Methods 77:109-114

23. Kuczewski A, Orsel K, Barkema HW, Kelton DF, Hutchins WA, van der Meer F (2017) Short communication: Evaluation of 5 different ELISA for the detection of bovine leukemia virus antibodies. J Dairy Sci 101:2433-2437

24. Larsen A, Gonzalez ET, Serena MS, Echeverría MG, Mortola E (2013) Expression of p24 gag protein of Bovine leukemia virus in insect cells and its use in immunodetection of the disease. Mol Biotechnol 54:475-483

25. Mammerickx M, Portetelle D, Burny A, Leunen J (1980) Detection by immunodiffusion- and radioimmunoassay-tests of antibodies to bovine leukemia virus antigens in sera of experimentally infected sheep and cattle. Zentralbl Veterinarmed B 27:291-303

26. Mamoun RZ, Astier T, Guillemain B, Duplan JF (1983) Bovine lymphosarcoma: expression of BLV-related proteins in cultured cells. J Gen Virol 64(Pt 9):1895-1905

27. Mamoun RZ, Morisson M, Rebeyrotte N, Busetta B, Couez D, Kettmann R, Hospital M, Guillemain B (1990) Sequence variability of bovine leukemia virus env gene and its relevance to the structure and antigenicity of the glycoproteins. J Virol 64:4180-4188

28. Miller JM, van der Maaten MJ (1982) Bovine Leukosis-its importance to the dairy industry in the United States. J Dairy Sci 65:2194-2203

29. Miyasaka T, Takeshima SN, Jimba M, Matsumoto Y, Kobayashi N, Matsuhashi T, Sentsui H, Aida Y (2013) Identification of Bovine leukocyte antigen class II haplotypes associated with variations in Bovine leukemia virus proviral load in Japanese Black cattle. Tissue Antigens 81:72-82

30. Molloy JB, Walker PJ, Baldock FC, Rodwell BJ, Cowley JA (1990) An enzyme-linked immunosorbent assay for detection of Bovine leukaemia virus p24 antibody in cattle. J Virol Methods 28:47-57

31. Momtaz H, Hemmatzadeh F, Keyvanfar H (2008) Expression of Bovine leukemia virus p24 protein in bacterial cell. Pak J Biol Sci 11:2433-2437

32. Mosadeghi P, Zarnagh HH, Mohammad-Zadeh M, Salehi Moghaddam M (2015) High-level soluble expression and onestep purification of HTLV-I P19 protein in Escherichia coli by Fusion Expression. Iran J Allergy Asthma Immunol 14:624-632

33. Murakami K, Kobayashi S, Konishi M, Kameyama K, Yamamoto T, Tsutsui T (2011) The recent prevalence of Bovine leukemia virus (BLV) infection among Japanese cattle. Vet Microbiol 148:84-88

34. Nekouei O, VanLeeuwen J, Sanchez J, Kelton D, Tiwari A, Keefe G (2015) Herd-level risk factors for infection with Bovine leukemia virus in Canadian dairy herds. Prev Vet Med 119:105-113

35. Ohno A, Takeshima SN, Matsumoto Y, Aida Y (2015) Risk factors associated with increased Bovine leukemia virus proviral load in infected cattle in Japan from 2012 to 2014. Virus Res 210:283-290

36. Ohshima K, Okada K, Numakunai S, Kayano H, Goto T (1988) An eradication program without economic loss in a herd infected with Bovine leukemia virus (BLV). Nihon Juigaku Zasshi 50:1074-1078
37. OIE (2018) World animal health information database-version enzootic Bovine leucosis. In: Manual of diagnostic tests and vaccines for terrestrial animals 2018. Chapter 2.4.10. 1-12. World organisation for animal Health, Paris. http://www.oie. int/en/international-standard-setting/terrestrial-manual/acces s-online/

38. Ott SL, Johnson R, Wells SJ (2003) Association between Bovine-leukosis virus seroprevalence and herd-level productivity on US dairy farms. Prev Vet Med 61:249-262

39. Panei CJ, Takeshima SN, Omori T, Nunoya T, Davis WC, Ishizaki H, Matoba K, Aida Y (2013) Estimation of Bovine leukemia virus (BLV) proviral load harbored by lymphocyte subpopulations in BLV-infected cattle at the subclinical stage of enzootic bovine leucosis using BLV-CoCoMo-qPCR. BMC Vet Res 9:95

40. Polat M, Ohno A, Takeshima SN, Kim J, Kikuya M, Matsumoto Y, Mingala CN, Onuma M, Aida Y (2015) Detection and molecular characterization of Bovine leukemia virus in Philippine cattle. Arch Virol 160:285-296

41. Polat M, Moe HH, Shimogiri T, Moe KK, Takeshima SN, Aida Y (2017) The molecular epidemiological study of Bovine leukemia virus infection in Myanmar cattle. Arch Virol 162:425-437

42. Polat M, Takeshima SN, Hosomichi K, Kim J, Miyasaka T, Yamada K, Arainga M, Murakami T, Matsumoto Y, de la Barra Diaz V, Panei CJ, Gonzalez ET, Kanemaki M, Onuma M, Giovambattista G, Aida Y (2016) A new genotype of Bovine leukemia virus in South America identified by NGS-based whole genome sequencing and molecular evolutionary genetic analysis. Retrovirology 13:4

43. Polat M, Takeshima SN, Aida Y (2017) Epidemiology and genetic diversity of Bovine leukemia virus. Virol J 14:209

44. Radke K, Grossman D, Kidd LC (1990) Humoral immune response of experimentally infected sheep defines two early periods of Bovine leukemia virus replication. Microb Pathog 9:159-171

45. Rhodes JK, Pelzer KD, Johnson YJ (2003) Economic implications of bovine leukemia virus infection in mid-Atlantic dairy herds. J Am Vet Med Assoc 223:346-352

46. Schupbach J, Kalyanaraman VS (1989) Detection of high concentrations of HTLV-1 p24 and a novel gag precursor, p45, in serum immune complexes of a healthy seropositive individual. Int J Cancer 44:90-94

47. Simard C, Richardson S, Dixon P, Belanger C, Maxwell P (2000) Enzyme-linked immunosorbent assay for the diagnosis of Bovine leukosis: comparison with the agar gel immunodiffusion test approved by the Canadian Food Inspection Agency. Can J Vet Res 64:101-106

48. Speers D, Phillips P, Dyer J (2005) Combination assay detecting both human immunodeficiency virus (HIV) p24 antigen and anti-HIV antibodies opens a second diagnostic window. J Clin Microbiol 43:5397-5399

49. Takeshima SN, Kitamura-Muramatsu Y, Yuan Y, Polat M, Saito S, Aida Y (2015) BLV-CoCoMo-qPCR-2: improvements to the BLV-CoCoMo-qPCR assay for Bovine leukemia virus by reducing primer degeneracy and constructing an optimal standard curve. Arch Virol 160:1325-1332

50. Telesnitsky A (2010) Retroviruses: molecular biology, genomics and pathogenesis. Future Virol 5:539-543

51. Toedter G, Pearlman S, Hofheinz D, Blakeslee J, Cockerell G, Dezzutti C, Yee J, Lal RB, Lairmore M (1992) Development of a monoclonal antibody-based p24 capsid antigen detection assay for HTLV-I, HTLV-II, and STLV-I infection. AIDS Res Hum Retroviruses 8:527-532

52. Uckert W, Grofova M, Beaudreau G (1984) Translational order of Bovine leukemia virus gag and env gene-coded proteins. Virology 135:288-292 
53. Ungar-Waron H, Brenner J, Paz R, Trainin Z (1992) Circulating immune complexes in Bovine leukemia virus (BLV)-infected cattle. Vet Immunol Immunopathol 34:173-179

54. Weiland F, Ueberschar S (1976) Ultrastructural comparison of Bovine leukemia virus (BLV) with C-type particles of other species. Arch Virol 52:187-190

55. Yang Y, Fan W, Mao Y, Yang Z, Lu G, Zhang R, Zhang H, Szeto C, Wang C (2016) Bovine leukemia virus infection in cattle of
China: association with reduced milk production and increased somatic cell score. J Dairy Sci 99:3688-3697

56. Yuan Y, Kitamura-Muramatsu Y, Saito S, Ishizaki H, Nakano M, Haga S, Matoba K, Ohno A, Murakami H, Takeshima SN, Aida $Y$ (2015) Detection of the BLV provirus from nasal secretion and saliva samples using BLV-CoCoMo-qPCR-2: comparison with blood samples from the same cattle. Virus Res 210:248-254 